\title{
An Analysis of the Correlation Between Clinical Indexes and Pathological Classifications in 202 Patients with Lupus Nephritis
}

\author{
Bang-Pin Pan ${ }^{1}, *$ \\ Zhi-Jian Feng ${ }^{2, *}$ \\ Xiao-Lan $\mathrm{Li}^{1}$ \\ Li-Ping $\mathrm{He}^{3}$ \\ Dong Jiang' \\ Rui-Xian Zhang' \\ Wen-Ting Cao' \\ Mei Cai ${ }^{1}$ \\ Yun-Feng $\mathrm{Bi}^{\prime}$ \\ Wen-Fang Wang' \\ Chun-Yan Luan'
}

'Department of Dermatology, The Second Affiliated Hospital of Kunming Medical University, Kunming, People's Republic of China; ${ }^{2}$ Department of Nephrology, The Second Affiliated Hospital of Kunming Medical University, Branch of National Clinical Research Center For Kidney Disease, Kunming, People's Republic of China; ${ }^{3}$ Department of Epidemiology and Health Statistics, School of Public Health, Kunming Medical University, Kunming, People's Republic of China

*These authors contributed equally to this work

\begin{abstract}
Objective: To investigate the correlation between clinical indexes and pathological classifications in 202 patients with lupus nephritis (LN).

Methods: A total of $202 \mathrm{LN}$ cases were retrospectively analyzed. All these patients met the four diagnostic criteria for systemic lupus erythematosus (SLE) of the American College of Rheumatology revised in 1997. The pathological diagnostic criteria of LN were in accordance with the pathological LN classification revised by the International Society of Nephrology and the Society of Kidney Pathology in 2003. The patients were scored according to the improved SLE Disease Activity Index 2000 (SLEDAI-2K), and their basic data, clinical data, laboratory data, and pathological data were collected.
\end{abstract}

Results: Among the 202 patients, the ratio of male to female was 1:5.73, and type IV was the most common pathological LN classification. There were differences in the urine analysis, hypertension incidence, blood cell analysis, blood lipids, renal function, plasma albumin, immunological indexes, renal pathological score among the different pathological types $(P<0.05)$. In the early finding of renal function damage of the patients, cystatin $\mathrm{C}$ sensitivity was significantly higher than that of serum creatinine and blood urea nitrogen. Multiple linear regression analysis show that there are strong correlations between $\mathrm{AI}$ and SLEDAI, 24hU-Pr, serum C3, serum ALB, BUN, creatinine, UA and PLT $(P<0.001)$; and there are correlations between $\mathrm{AI}$ and serum IgM, IgA, C4, TC and LDL-C $(P<0.05)$.

Conclusion: There is a clear correlation between pathological classifications and clinical indexes of LN.

Trial Registration: Shen-PJ-2018-40, Study on Clinical and Molecular Mechanism of SLE.

Keywords: lupus nephritis, kidney pathology, clinical indexes

\section{Background}

Systemic Lupus Erythematosus (SLE) is an autoimmune disease in which multiple systems and organs are damaged throughout the body. The exact cause of SLE is not yet clear, but according to current research and analysis, it is mainly related to genetic susceptibility, environmental risk factors and sex hormones. Lupus nephritis (LN) is the most common, serious and potential visceral damage that may progress to renal failure. ${ }^{1,2}$ SLE is the most common systemic autoimmune disease in China, with a population incidence of 30.13 to 70.41 per 100,000 people, and $40 \%$ to $60 \%$ of SLE patients have lupus nephritis at the beginning of the disease. ${ }^{3} \mathrm{LN}$ is mainly caused by kidney damage caused by circulating or in situ immune complex
Correspondence: Xiao-Lan Li

Tel +8687163402212

Fax +8687165334416

Email lixiaolan880923@I63.com 
deposition; a small part of SLE damages the kidney through non-immune complex pathways (such as lupus interstitial nephritis) or renal vascular disease. ${ }^{3}$ Among which IC deposition is currently recognized as core link, which process mainly activates the classical pathway of the complement system, induces kidney tissue cell damage and local secretion of inflammatory cytokines and chemokines. At the same time, where IC is deposited in the kidney, the immune system responds to IC and causes local inflammatory damage to a large extent determine the pathological type and clinical results of each patient's LN. ${ }^{4}$

The cumulative incidence of $\mathrm{LN}$ is highest in Asians (55\%), followed by African Americans (50.5\%), Hispanics (43\%), and Caucasians (14\%). Other continents are relatively low. Up to $25 \%$ of $\mathrm{LN}$ patients develop end-stage renal disease (ESRD) after about 10 years of renal damage. ${ }^{5}$ In China, nearly half of SLE patients have LN, which is higher than that of Caucasians. $\mathrm{LN}$ is the most common secondary immune glomerular disease in Chinese with $81 \% \sim 98 \%$ of 10 -year renal survival rate, and it is one of the most common causes of ESRD, also an important cause of death in SLE patients. $^{3}$

Patients with SLE with any sign of kidney involvement (glomerular haematuria and/or cellular casts, proteinuria $>0.5 \mathrm{~g} / 24$ hours (or spot urine protein-to-creatine ratio (UPCR) $>500 \mathrm{mg} / \mathrm{g}$ ), unexplained decrease in glomerular filtration rate (GFR)) are candidates for kidney biopsy. Mild clinical presentations (eg, subnephrotic proteinuria) can nonetheless be associated with active histological lesions. ${ }^{5}$ In a review of kidney biopsies performed during 1970-2016, earlier use of biopsy based on urinary abnormalities, as done from 2001 to 2016, was associated with improved outcomes, despite similar rates of severe histology. ${ }^{5}$

However, renal biopsy is difficult to popularize in underdeveloped areas in western China. On the one hand, renal biopsy is an invasive examination requiring high operation and laboratory technologies, but with poor patient acceptance and contraindications to puncture. On the other hand, the different kidney pathological types can be transformed into each other for most patients in their long course of the disease, so they have to accept repeat renal biopsies, which brings increased bleeding risks and higher medical costs. At present, the kidney disease progression assessment and treatment plan of nearly two thirds of LN patients are still comprehensively analyzed and evaluated based on their clinical manifestations, laboratory indicators and clinician experience even in our hospital which is the provincial center of Lupus Erythematosus and Immune Skin Disease Clinic. In recent years, due to the participation of third-party testing companies, this situation has been improved just a little.

This study retrospectively analyzed and summarized the characteristics of the clinical indicators and renal pathological typing of $202 \mathrm{LN}$ patients in Yunnan, attempted to find the association between renal pathological types, AI scores with SLEDAI scores, clinical features and laboratory indicators in LN patients, to explore a relatively accurate and sensitive assessment standards of kidney damage insted of biopsy, finally to develop a personalized and appropriate treatment plan for $\mathrm{LN}$ patients who are unaccessable to renal biopsy pathology temporarily or in long term.

\section{Patients and Methods}

\section{Patients}

A total of $202 \mathrm{LN}$ cases, diagnosed via renal puncture biopsy in the departments of Dermatology, Rheumatology and Nephrology at the second affiliated hospital of Kunming Medical University between April 2007 and November 2018, were included. All the patients met the four diagnostic criteria for SLE of the American College of Rheumatology revised in $1997 .^{2}$ The pathological diagnostic criteria of LN were in accordance with the pathological LN classification revised by the International Society of Nephrology and the Society of Kidney Pathology in 2003, ${ }^{3}$ and we adopted the revision of kidney tissue activity index (AI) and chronic index (CI) scoring standards from National Institutes of Health (NIH). ${ }^{3}$ All patients were scored according to the improved SLE Disease Activity Index 2000 (SLEDAI$2 \mathrm{~K}$ ), ${ }^{4}$ and their basic data, clinical data (ANA, A-dsDNA, ENA enzyme spectrum, serum immunoglobulin + complement assay, urine biochemistry, renal function, 24-hour urine protein quantification, endogenous creatinine clearance rate, and urine microprotein assay), laboratory data, and pathological data were collected. The current study was approved by the Ethics Committee of our hospital, and all patients signed written informed consent. Pediatric patients were excluded.

A biopsy is a common procedure for establishing the extent of a disease and the indications for renal failure are as follows: in patients with LN, 24-hour quantitative urinary protein $>2.0 \mathrm{~g} / 24 \mathrm{~h}$ is a symptom of nephrotic syndrome 
and serum complement C3 lower than normal shows there is insufficient renal function. However, it should be noted that about two-thirds of $\mathrm{LN}$ patients with proteinuria do not undergo a renal biopsy because the patients and their families have concerns about "kidney injury or bleeding" caused by puncture exacerbating their condition and do not agree to be tested. (unpublished observation/personal experience). The diagnosis and AI /CI scoring of LN was performed by qualified pathologists from the Department of Pathology and Dermatology of the Second Affiliated Hospital of Kunming Medical University based on routine HE staining of the kidney and direct immunofluorescence (DIF). A small number of patients were also examined by electron microscopy and completed by a professional institution.

\section{Methods}

The quantitative data conforming to the normal distribution were expressed by $\overline{\mathrm{X}} \pm \mathrm{S}$. The comparison between groups was conducted via a $t$-test (between two groups), one-way ANOVA (more than 2 groups), $\chi 2$ test, or Fisher's exact probability method, and the qualitative data were expressed by rate. The relationship between the 24-h urinary protein level and blood lipid level was analyzed by multiple linear regression analysis.

\section{Results}

\section{Basic Information}

Of the 202 patients, 172 were female $(85.15 \%)$, and 30 were male $(14.85 \%)$. Thus, the ratio of men to women was 1:5.73. The onset age of the disease was between 8 and 69 years old, and the average onset age was 27.96 years old. The duration of the disease was $2-426$ months, with an average of 114.69 months. One patient died two months after onset and another one four months afterwards.

All 202 patients received long-term or lifelong drug treatment with corticosteroids, hydroxychloroquine and immunosuppressants, but none used retinoids and other medicines that could lead to elevated triglycerides (TG).

\section{Proportion of Different Pathological Types of Kidney Disease}

Type IV was the most common $(\mathrm{n}=117)$, followed by types II $(\mathrm{n}=25), \mathrm{V}+\mathrm{IV}(\mathrm{n}=17)$, III $(\mathrm{n}=16), \mathrm{V}+\mathrm{III}(\mathrm{n}=$ $12), \mathrm{V}(\mathrm{n}=11)$, and VI $(\mathrm{n}=4)$. There were no complex types of type I. Type IV was the most common type in both the male and female patients, and there was no significant difference in the distribution of gender among the seven pathological types $(P>0.05)$.

\section{Routine Urine Examination and Incidence of Anemia and Hypertension}

Types II and III were regarded as mild LN and type IV as severe LN. The incidence of renal hematuria in severe LN was significantly higher than that in mild LN $(P<0.001)$, and the incidence of aseptic pyuria in type II was significantly lower than that in the other types $(P<0.001)$. The incidence of anemia among the different pathological types showed very little difference $(P=0.047)$ and was highest in types VI, IV, III, and V+IV. The incidence of hypertension in type II was significantly lower than that in type $\mathrm{V}+\mathrm{IV}(P=0.004)$. There was no difference in the incidence of cylindruria among the different pathological types $(P>0.05)$ (see Table 1$)$.

Table I Comparison of Laboratory Indexes of Each Pathological Type of LN [Number of Cases (\%)]

\begin{tabular}{|c|c|c|c|c|c|}
\hline Clinical Index & Anemia (\%) & Renal Hematuria (\%) & Aseptic Pyuria & Cylindruria & Hypertension \\
\hline$\|(n=25)$ & $9(36.0)$ & $5(20.0)$ & $5(20.0)$ & $\mathrm{I}(4.0)$ & $4(16.4)$ \\
\hline III $(n=16)$ & $10(62.5)$ & $7(43.8)$ & $\mathrm{II}(68.8)^{\wedge}$ & $I(6.3)$ & $7(43.8)$ \\
\hline $\operatorname{IV}(n=117)$ & $56(47.9)$ & $96(82.1)^{\wedge \bullet}$ & $82(70.1)^{\wedge}$ & $10(8.5)$ & $5 I(43.6)$ \\
\hline$V(n=I I)$ & $5(45.5)$ & $8(72.7)$ & $4(36.4)$ & $0(0.0)$ & $4(36.4)$ \\
\hline $\operatorname{VI}(n=4)$ & $4(100.0)$ & $3(75.0)$ & $2(50.0)$ & $0(0.0)$ & $3(75.00)$ \\
\hline$V+I I I(n=I 2)$ & $2(16.7)$ & $10(83.3)$ & $8(66.7)$ & $I(8.3)$ & $7(58.3)$ \\
\hline$V+I V(n=I 7)$ & $10(58.8)$ & $\mid 4(82.4)^{\wedge}$ & $12(70.6)^{\wedge}$ & $0(0.0)$ & $13(76.5)^{\Lambda}$ \\
\hline Total $(n=202)$ & $96(47.52)$ & I43(70.79) & $\mid 24(6 \mid .39)$ & $13(6.44)$ & $89(44.06)$ \\
\hline$P$-value & 0.047 & $<0.001$ & $<0.001$ & 0.892 & 0.004 \\
\hline
\end{tabular}

Notes: ${ }^{\Delta}$ Compared with type II LN, $P<0.05$ and ${ }^{\bullet}$ Compared with type III LN, $P<0.05$. 


\section{Blood Cell Analysis}

The blood cell analysis results showed there were differences in the platelet (PLT) value among the different pathological types $(P=0.024)$. Type $\mathrm{V}+\mathrm{IV}$ had the lowest value, significantly lower than those of types II, IV, and V; type $\mathrm{V}$ had the highest value, significantly higher than that of types III, IV, and V+IV. There was no significant difference in the leukocyte and lymphocyte values among the different pathological types $(P>0.05)$ (see Figure 1$)$.

\section{Blood Lipid Test}

The total cholesterol (TC) and low-density lipoprotein cholesterol (LDLC) values of severe LN were significantly higher than those of mild LN. The dyslipidemia of type $\mathrm{V}$ was particularly prominent. The TC and LDLC values of type $\mathrm{V}$ were significantly higher than those of types II, III, IV, and VI, while the LDLC values of types V + III were significantly higher than those of types II, III, and VI. However, there was no significant difference in the triglyceride (TG) or high-density lipoprotein cholesterol value between the different pathological types $(P>0.05)$ (see Figure 2).

\section{Renal Function Examination}

\section{Routine Renal Function Index}

The renal function of each pathological type was damaged to different degrees. While the renal insufficiencies of types $\mathrm{IV}, \mathrm{V}+\mathrm{III}, \mathrm{V}+\mathrm{IV}$, and VI were serious, those of type VI were the worst. There was a significant difference in the serum creatine (Scr) and blood urea nitrogen (BUN) values between the different pathological types $(P<0.001, P<$ 0.001). The Scr and BUN values of type VI were significantly higher than those of the other types, and the BUN value of type IV was significantly higher than that of types II and V. Moreover, there were differences in the UA value among the different pathological types $(P=0.018)$. The UA value in types IV, VI, and V+III was significantly higher than in type II, while that in type VI was significantly higher than in types II, III, and V. Each phathological type group has different degrees of albuminuria and hypoalbuminemia which is manifested as significant differences in 24-h urinary protein quantification (24hU-Pr) and serum albumin (ALB) values $(P<0.001, P<0.001)$. The $24 \mathrm{hU}-\mathrm{Pr}$ values of types IV, V, and compound $\mathrm{V}(\mathrm{V}+\mathrm{III}$ and $\mathrm{V}+\mathrm{IV})$ were significantly higher than those of types II and III. The ALB value of types IV, V, and compound $\mathrm{V}$ was significantly lower than that of types II and III; the value of type V+III was the lowest, significantly lower than that of types II, III, and IV (see Figure 3).

\section{Comparison of Cystatin C and Conventional Renal Function Indexes}

With regard to judging renal function damage in patients with LN, 63 of the 202 patients underwent a cystatin $\mathrm{C}$ (Cys-C) test, wherein Cys-C $>1.03 \mathrm{mg} / \mathrm{L}, \mathrm{Scr}>115 \mu$ $\mathrm{mol} / \mathrm{L}$, and $\mathrm{BUN}>7.2 \mathrm{mmol} / \mathrm{L}$ was judged as renal dysfunction. The sensitivities of Cys-C, Scr, and BUN were $88.89 \%, 25.40 \%$, and $41.27 \%$, respectively. The sensitivity of renal function damage in patients with $\mathrm{LN}$ diagnosed by Cys-C was significantly higher than that in patients diagnosed via $\mathrm{Scr}$ and $\mathrm{BUN}(P<0.001, P<$ $0.001)$, which indicated that Cys-C is more sensitive than Scr and BUN in the diagnosis of renal function damage in such patients (see Figure 3).

\section{Immunologic Test}

The highest rate of anti-ds-DNA antibody was $70.59 \%$ in type $\mathrm{V}+\mathrm{IV}$, followed by $52.41 \%$ in type IV. The positive rate of anti-ds-DNA antibody in types IV and V+IV was significantly higher than that in type II. There were no significant differences in the positive rate of ANA, anti-Smith antibody,
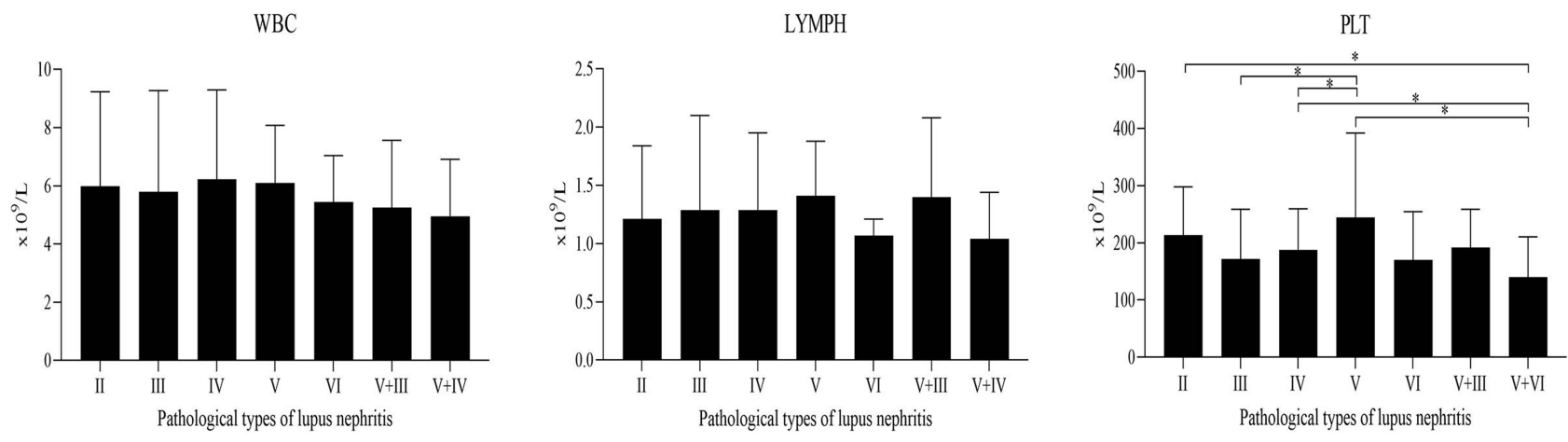

Figure I Comparison of blood cell counts of each pathological type of LN $(* P<0.05)$. 

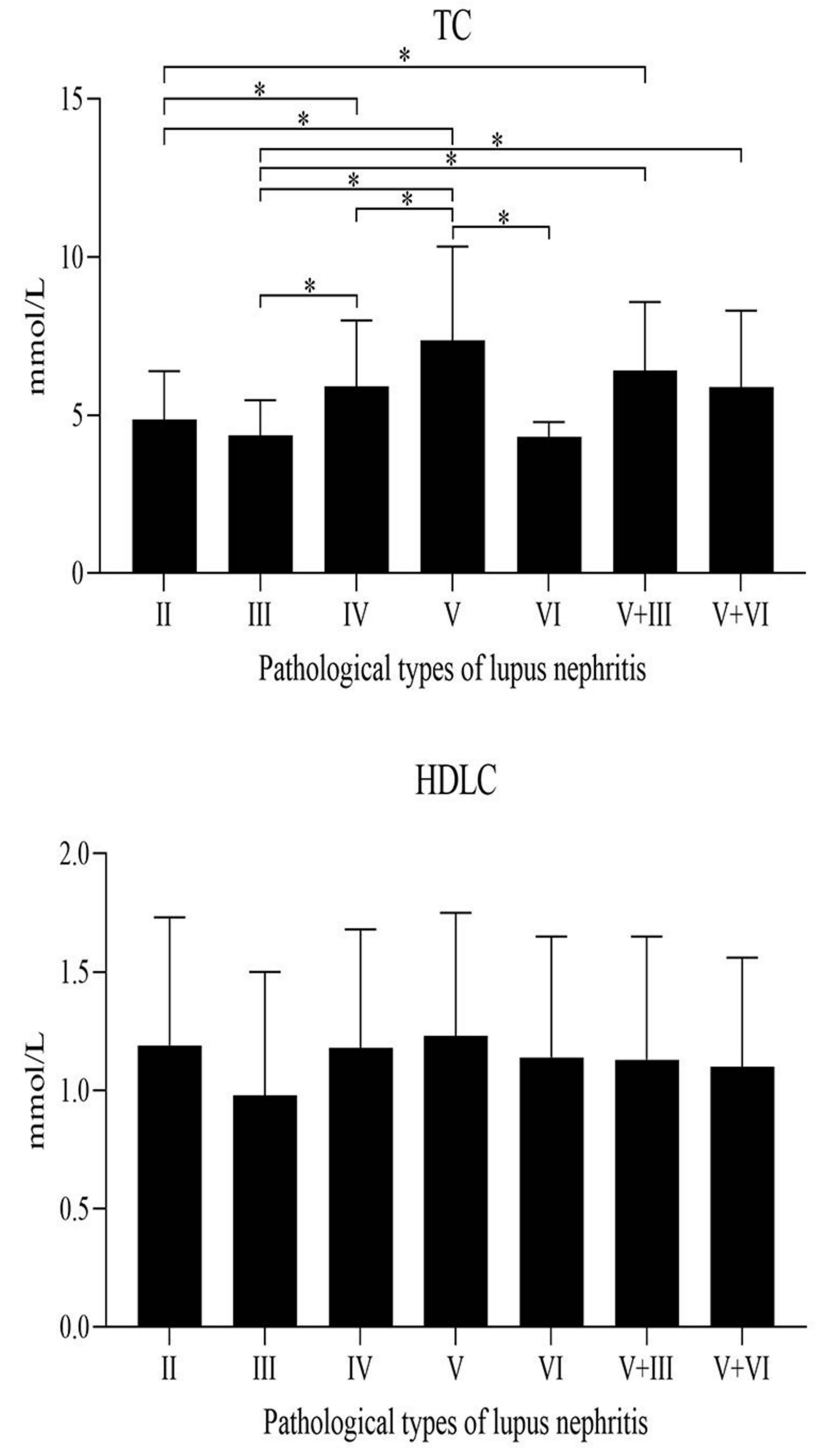

Figure 2 Comparison of blood lipid test of each pathological type of LN $(* P<0.05)$.

anti-SSA antibody, anti-Ro52 antibody, anti-SSB antibody, anti-PCNA antibody, ANuA, AHA, ARPA, and ANCA among the different pathological types $(P>0.05)$. The complement $\mathrm{C} 3$ values of each pathological type decreased to varying degrees. The complement $\mathrm{C} 3$ and $\mathrm{C} 4$ values of type V+IV were the lowest. There were differences in the complement $\mathrm{C} 3$ level among the different pathological types $(P=0.021)$, among which the level of type $\mathrm{V}+\mathrm{IV}$ was significantly lower than that of types II, IV, V, and VI, and the level of type IV significantly lower than that of type II. There were also differences in the complement $\mathrm{C} 4$ level among the different pathological types $(P=0.038)$. Types IV and V + IV had a significantly lower level than types $\mathrm{V}$ and VI. However, there was no significant difference in
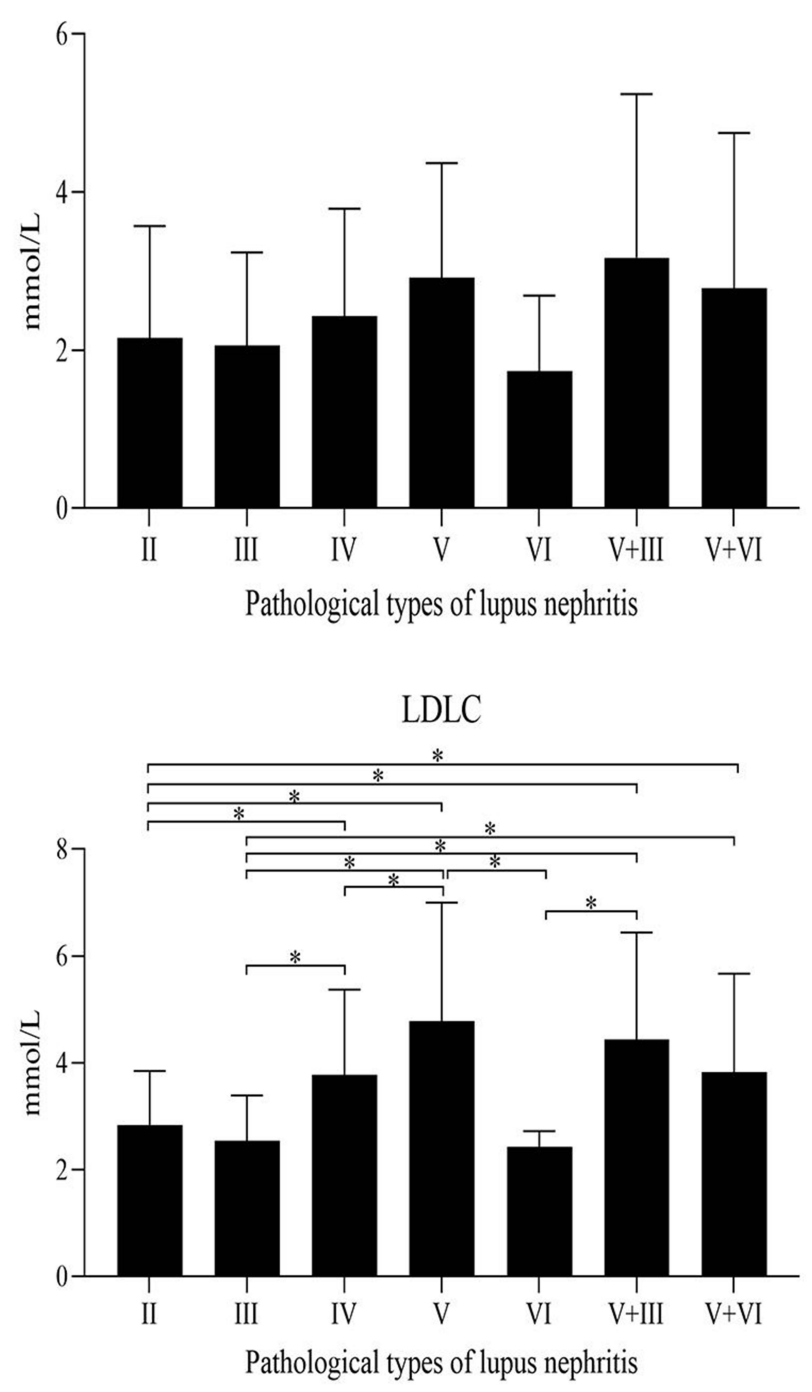

the immunoglobulin (Ig) G, IgA, or IgM among the different pathological types $(P>0.05)$ (see Figure 4).

\section{Pathological Score $(\mathrm{Al} / \mathrm{Cl})$ and SLEDAI-2K Score}

The chronicity index (CI) value of type VI was the highest and those of types II and III the lowest. There were significant differences in the AI value among the different pathological types $(P<0.001)$, that of type II being significantly lower than those of the others, and those of types IV and V +IV being significantly higher than that of types II, III, V, and $\mathrm{V}+\mathrm{III}$. In addition, there were significant differences in the CI value among the different pathological types $(P<$ 0.001), type II being significantly lower than the others and 
CREA

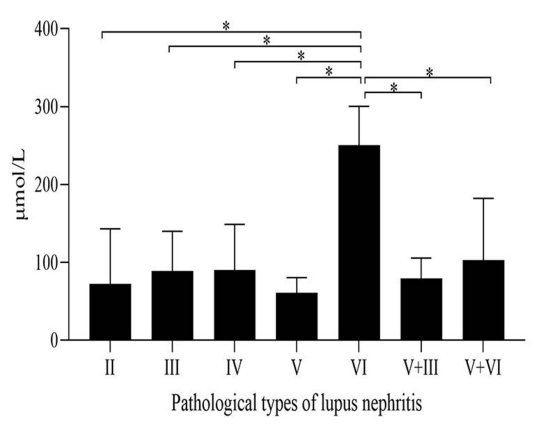

24hU-Pr

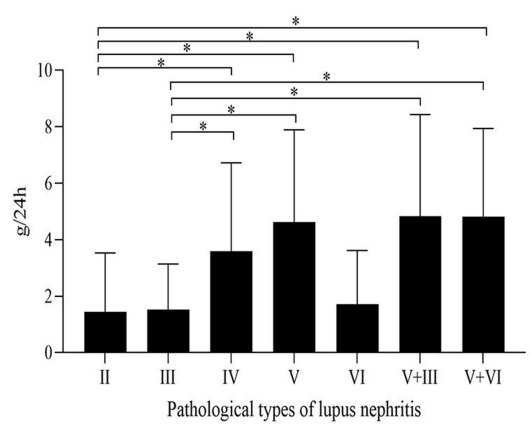

UREA

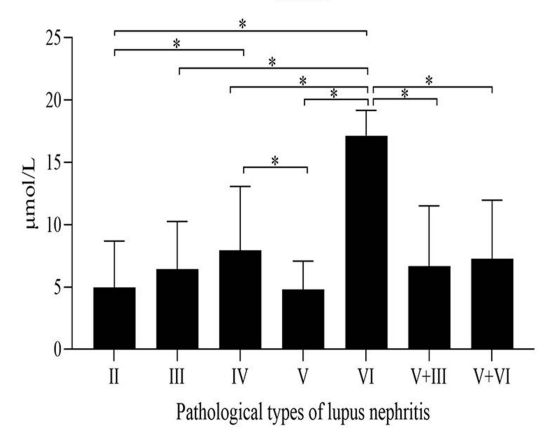

ALB

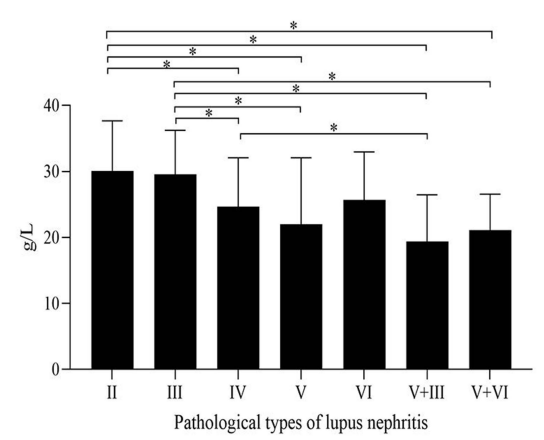

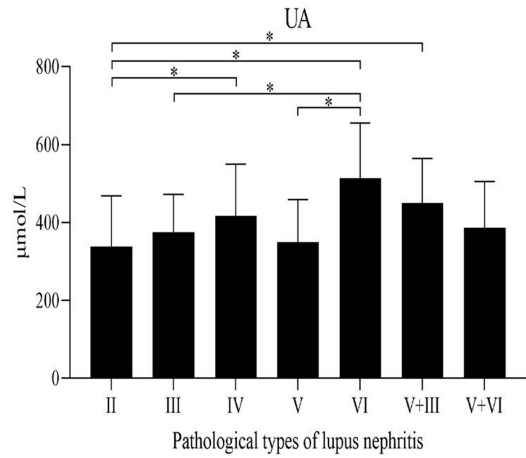

Cys-C

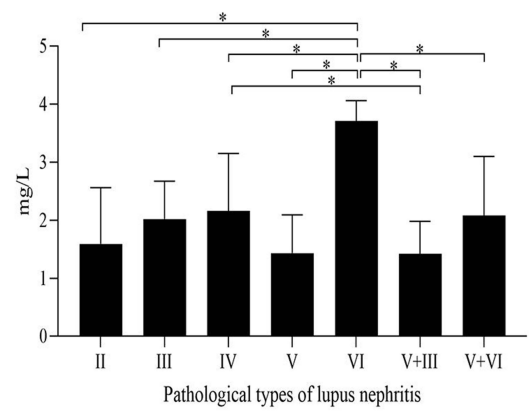

Figure 3 Comparison of routine renal function index of each pathological type of LN $(* P<0.05)$.

type VI significantly higher. The SLEDAI-2K scores of that of type II being the lowest. There was a significant types III, IV, V+III, and V+IV were higher than that of difference in the SLEDAI-2K score among the different type II and the score of type V + IV was the highest, with pathological types $(P<0.001)$. The scores of types III, IV,

$\lg G$

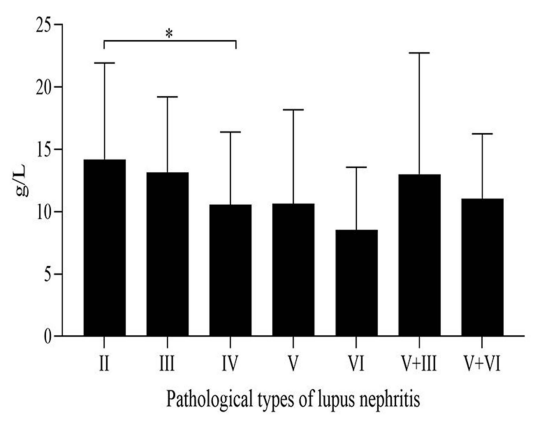

$\mathrm{C} 3$

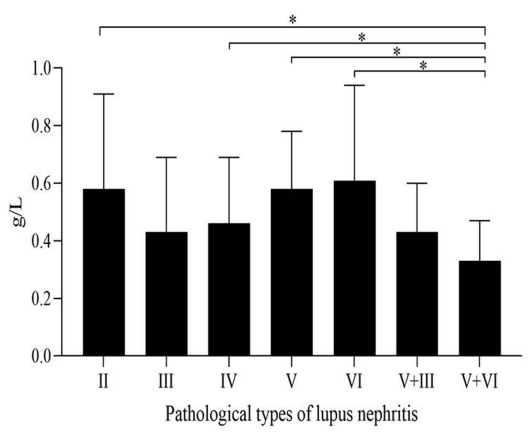

$\operatorname{IgA}$
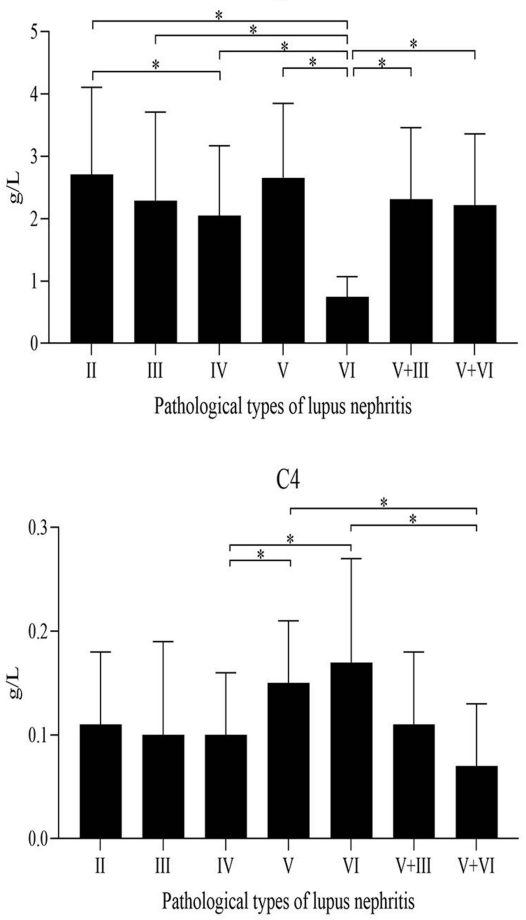

$\operatorname{IgM}$

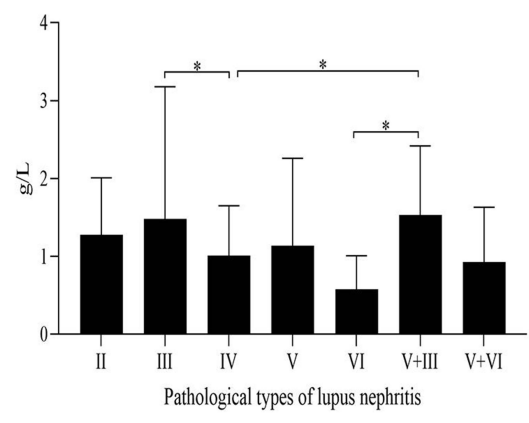

Figure 4 Comparison of immunologic test of each pathological type of LN $(* P<0.05)$. 

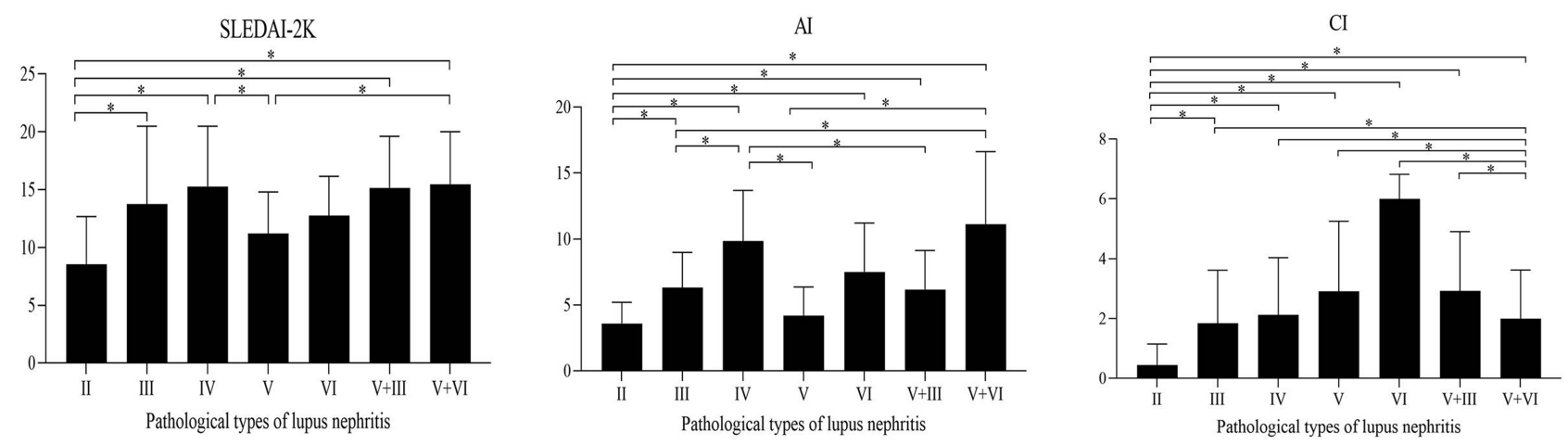

Figure 5 Comparison of pathological score $(\mathrm{Al} / \mathrm{Cl})$ and SLEDAI-2K score of each pathological type of LN $(* P<0.05)$.

$\mathrm{V}+\mathrm{III}$, and $\mathrm{V}+\mathrm{IV}$ were significantly higher than that of type II, while the score of type $\mathrm{V}$ was significantly lower than those of types IV and V+IV (see Figure 5).

\section{Multiple Linear Regression Analysis of Pathological Score Al and Various Clinical and Laboratory Indicators}

The results of multiple linear regression analysis show that there are strong correlations between AI and SLEDAI, 24hU-Pr, serum C3, serum ALB, BUN, creatinine, UA and PLT $(P<0.001)$ (see Figure 6).

The results of multiple linear regression analysis show that there are correlations between $\mathrm{AI}$ and serum $\operatorname{IgM}$, IgA, C4, TC and LDL-C $(P<0.05)$ (see Figure 7).

\section{Discussion}

Lupus nephritis is one of the most common and serious visceral complications in SLE. Immunohistochemical and electron microscopic examinations have shown that renal damage in patients with SLE is as high as $100 \% .^{6}$ In this study, the ratio of male to female patients with $\mathrm{LN}$ was 1:5.73, which is within the scope of other Chinese research (1:5.5 9.7), ${ }^{7-9}$ and type IV was the most common pathology, also similar to other domestic research. ${ }^{10,11}$

This study showed that the incidence of hematuria under microscope be the most common (143 cases, $70.79 \%$ ), consistent with Jiang Kun's. ${ }^{12}$ Compared with the mild types, types IV, $\mathrm{V}$, and compound $\mathrm{V}$ had higher red blood cell and white blood cell counts and ALB, which is filtered from the glomerulus filtration barrier. A higher incidence of renal hematuria and aseptic pyuria, higher quantity of 24hU-Pr, and lower ALB levels often occurred in the severe types. A previous case-control study on patients with SLE showed that anemia is often combined with other types of blood cell reduction, hypocomplementemia, a higher positive rate of anti-ds-DNA antibody, and a higher risk of renal injury. ${ }^{13}$ In this study, severe anemia and low PLT count occurred primarily in the severe types. The heavier the renal damage, the more severe the anemia, the lower the PLT count, and the higher the SLEDAI-2K score in types IV and compound V. The data also showed that the TC and LDLC levels in severe LN were significantly higher than those in mild LN. Hyperlipemia was particularly prominent in type V. It was suggested that dyslipidemia in patients with SLE is significantly correlated with the occurrence of LN. Abnormal elevation of TC and LDLC levels would appear to an independent risk factor for renal damage in such patients.

In some patients, a decrease in GFR did not cause Scr change at the early stage of $\mathrm{LN}$, even in the case of a $50 \%$ decrease, ie, a "creatinine-blind area". The Cys-C was not affected by creatinine-blind areas, could sensitively reflect early GFR changes, and was beneficial for the early evaluation of disease and treatment. ${ }^{14}$ The abnormal rate of renal function damage in patients with $\mathrm{LN}$ diagnosed by Cys-C was significantly higher than that diagnosed by Scr and BUN $(P<0.001, P<0.001)$. This suggested that the Cys-C test is faster and more convenient in the detection of minimal renal function injury in patients with early LN and can detect renal function changes in such patients, allowing for early diagnosis and treatment and, thereby, an improvement in prognosis.

Many studies from different countries have confirmed that the deposition of anti-ds-DNA, ANuA, and ANCA antibodies in the kidneys plays a key role in the pathogenesis, progression, and severity of LN as well as the diagnosis, treatment, and prognosis of the disease. However, it remains unclear whether there are differences in the kind and titer of autoantibodies in the different pathological 

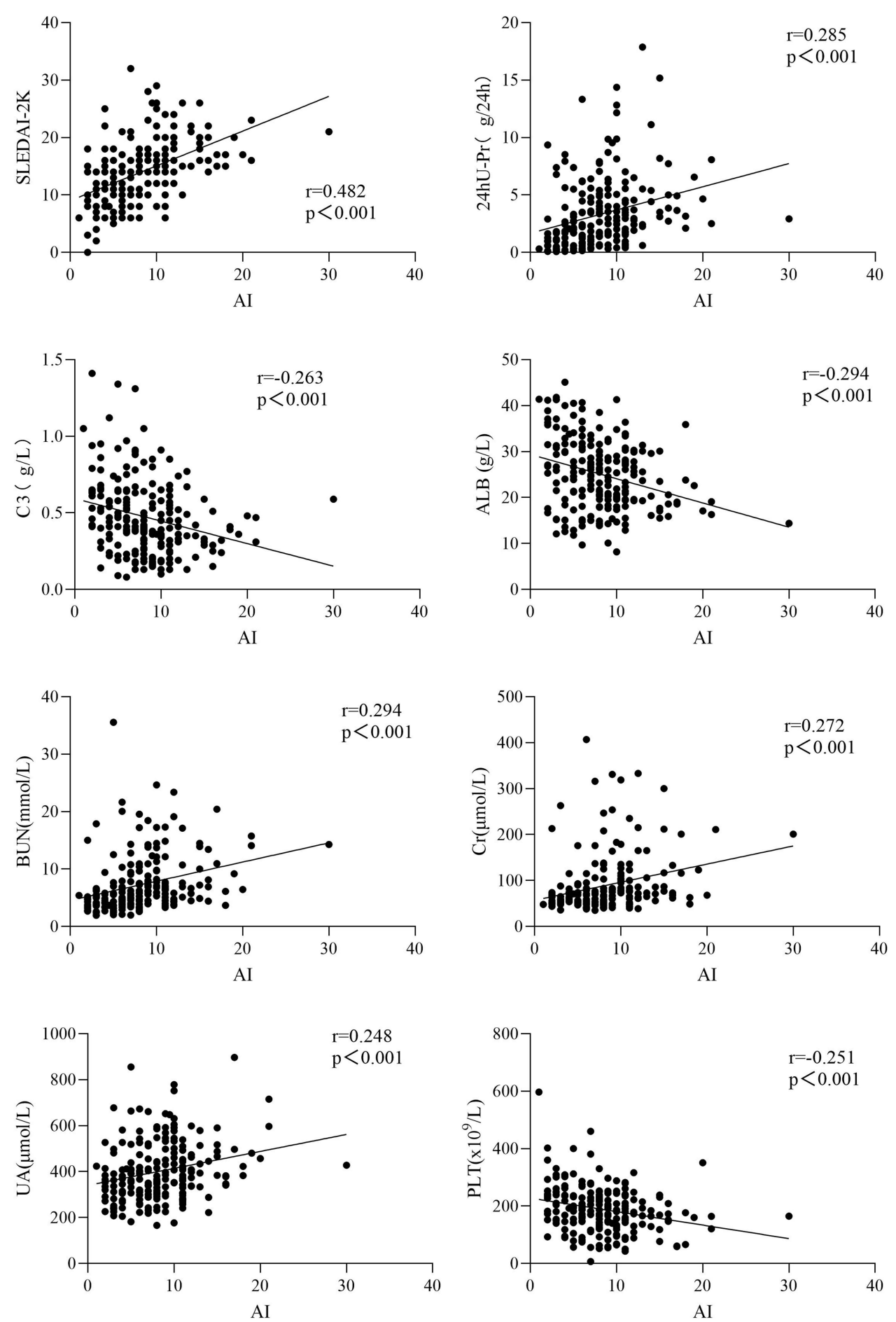

Figure 6 Multiple linear regression analysis of pathological score Al and SLEDAI, 24hU-Pr, C3, ALB, BUN, Scr, UA and PLT (P < 0.00I).

types of LN. In this study, the positive rate of anti-dsDNA antibody was significantly higher in types IV and V + IV than in type II $(P<0.05)$. The results also showed that anti-ds-DNA antibody was related to the degree of renal lesion, which was consistent with most research. ${ }^{15,16}$ Previous studies have shown that the level of complement
C3 is lower in patients with LN with normal renal function and no clinical manifestations of renal damage diagnosed by renal biopsy than in patients with SLE without $\mathrm{LN}$, indicating that a low level of complement $\mathrm{C} 3$ is a predictor of silent $\mathrm{LN}^{17}$ Herein, we found that the complement $\mathrm{C} 3$ level significantly differed among the 

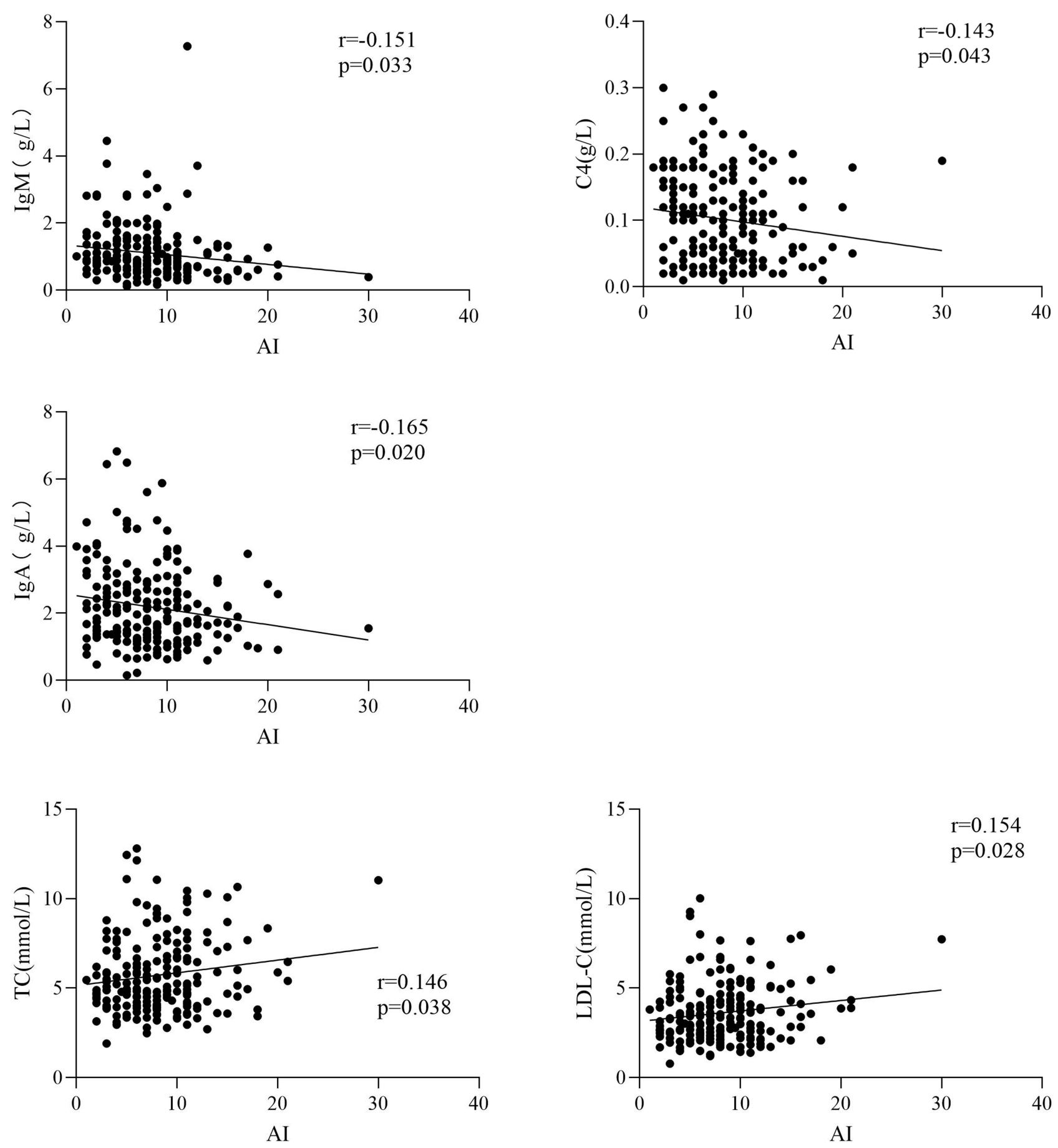

Figure 7 Multiple linear regression analysis of pathological score of $\lg M, \lg A, C 4, T C$ and LDL-C $(P<0.05)$.

different pathological disease types; the levels in types IV and V+IV were especially low. Therefore, a low level of complement C3 suggests that patients with SLE have renal involvement.

The pathological changes of LN can be divided into active and chronic. High AI score was an indication of active immunosuppressive therapy, ie, "induction remission". If the treatment was given actively, its pathological degree was possibly reversible, and the lifespan of the patient was prolonged. In contrast, the CI level determined the irreversible degree of lesions and long-term renal function: the higher the $\mathrm{CI}$, the worse the prognosis. This was roughly consistent with Yan Zhizhen's ${ }^{10}$ research results. However, some laboratory indexes 
might be affected by factors apart from renal involvement, but since we had no longitudinal data (before and after biopsy), it was not possible to investigate such factors in this study. This issue needs to be addressed in the future. In addition, the current consensus is that histology cannot be reliably predicted by clinical data alone, and, thus, biopsy findings are the recommended basis for therapeutic decisions. The pathological score of LN includes AI and CI. The treatment of LN needs to be formulated according to the pathological score. In this study, the results of multiple linear regression analysis show that there are strong correlations between $\mathrm{AI}$ and SLEDAI, 24hU-Pr, serum C3, ALB, BUN, Scr, UA and PLT $(P<0.001)$, and there are correlations between AI and serum IgM, IgA, C4, TC and LDL-C $(P<0.05)$. Therefore, when histology is not available, various clinical markers/indicators could guide decision-making of therapeutic plan to a certain degree.

\section{Conclusions}

The results of this study suggest that there is a clear correlation between pathological classifications, renal AI scores and clinical indexes of $\mathrm{LN}$, which may be of guiding value for early diagnosis, pathological classification, early treatment, and prognosis of the disease.

\section{Data Sharing Statement}

The datasets used and/or analysed during the current study are available from the corresponding author on reasonable request.

\section{Ethics Approval and Consent to Participate}

The research has been approved by ethics committee of the Second Affiliated Hospital of Kunming Medical University. This study was conducted in accordance with the declaration of Helsinki. Written informed consent was obtained from all participants.

\section{Consent for Publication}

The institutional consent form Department of Dermatology, The Second Affiliated Hospital of Kunming Medical University have been given to all participants and received.

\section{Acknowledgments}

The authors would like to acknowledge the Department of Histopathology, Clinical Laboratory of the Second
Affiliated Hospital of Kunming Medical University and the People's Hospital of Wenshan Prefectur for supporting this research. Dr Bang-Pin Pan is now also affiliated with Department of Dermatology \& Rheumatology Immunology, The People's Hospital of Wenshan Prefecture, Yunnan, China.

\section{Funding}

Key Projects of Yunnan Provincial Science and Technology Plan [No.: 2017FE468 (-004)] and the National Natural Science Foundation of China (No.: 81360457).

\section{Disclosure}

The authors report no competing interests.

\section{References}

1. Balow JE, Iii HAA. Treatment of proliferative lupus nephritis. $A m$ J Kidney Dis. 2004;43(2):383-385. doi:10.1053/j.ajkd.2003.12.008

2. Mok CC. Prognostic factors in lupus nephritis. Lupus. 2005;14 (1):39-44. doi:10.1191/0961203305lu2057oa

3. Chinese lupus nephritis diagnosis and treatment guidelines compilation group. Chinese lupus nephritis diagnosis and treatment guidelines. Chin Med J. 2019;99(44):3441-3455.

4. Flores-mendoza G, Sansón SP, Rodríguez-castro S, Crispín JC, Rosetti F. Mechanisms of tissue injury in lupus nephritis. Trends Mol Med. 2018;24(4):364-378.

5. Fanouriakis A, Kostopoulou M. 2019 Update of the Joint European League Against Rheumatism and European Renal Association-European Dialysis and Transplant Association (EULAR/ERA-EDTA) recommendations for the management of lupus nephritis. Ann Rheum Dis. 2020;79 (6):713-723. doi:10.1136/annrheumdis-2020-216924

6. Yu X-Q, Wei J-L. Kidney disease in china: recent progress and prospects. Chin Med J. 2009;122(17):2048-2053.

7. Mei Z, Sheng-qian X, Jing C, et al. Relationship between renal pathological classifications and clinical parameters. Chin J General Pract. 2017;15(2):227-229, 242.

8. Wen-hui G. Immune Index and Pathological Types of Lupus Nephritis: Analysis of 97 Patients. Changchun: Jilin University; 2014.

9. Ming-hui Z, Yan-hui L, Xin-lan L, et al. Analysis of clinical and pathological of 144 patients with lupus nephritis. Guangdong Med J. 2009;30(3):453-456.

10. Zhou F-D, Zhao M-H, Zou Z, et al. The changing spectrum of primary glomerular diseases within 15 years: a survey of 3331 patients in a single Chinese centre. Nephrol Dial Transplant. 2009;24(3):870-876. doi:10.1093/ndt/gfn554

11. Zhi-zhen Y. The Correlation Analysis Between Clinical Score and Pathological Score in Patients with Lupus Nephritis. Changchun: Jilin University; 2018.

12. Kun J. The Correlation Between Pathological Classification, Clinical Manifestation and Laboratory Diagnosis of Lupus Nephritis. Shihezi: Shihezi University; 2016.

13. Zhuo-qing Q. A Case-Control Study of Clinical Feature and Prognosis of Systemic Lupus Erythematosus Patients with Anemia. Beijing: Chinese Academy of Medical Sciences; 2012.

14. Xiao-yan Z, Yu Y. Clinical application of cystatin $\mathrm{c}$ and its detection standardization progress. Labeled Immunoassays Clin Med. 2017;24 (6):717-720. 
15. Hanly G, Thompson K, McCurdy G, et al. Measurement of autoantibodies using multiplex methodology in patients with systemic lupus erythematosus. $J$ Immunol Methods. 2010;352(1):147-152. doi:10.1016/j.jim.2009.10.003

16. Yi Z. The Analysis of Clinical Characteristics and Pathological Type in Lupus Nephritis. Shanghai: Fudan University; 2010.
17. Ishizaki J, Saito K, Nawata M, et al. Low complements and high titre of anti-sm antibody as predictors of histopathologically proven silent lupus nephritis without abnormal urinalysis in patients with systemic lupus erythematosus. Rheumatology. 2015;54(3):405-412. doi:10.1093/rheumatology/keu343

\section{Publish your work in this journal}

The Journal of Inflammation Research is an international, peerreviewed open-access journal that welcomes laboratory and clinical findings on the molecular basis, cell biology and pharmacology of inflammation including original research, reviews, symposium reports, hypothesis formation and commentaries on: acute/chronic inflammation; mediators of inflammation; cellular processes; molecular mechanisms; pharmacology and novel anti-inflammatory drugs; clinical conditions involving inflammation. The manuscript management system is completely online and includes a very quick and fair peerreview system. Visit http://www.dovepress.com/testimonials.php to read real quotes from published authors.

Submit your manuscript here: https://www.dovepress.com/journal-of-inflammation-research-journal 